\title{
Incidence of metabolic syndrome among night-shift healthcare workers
}

\author{
A Pietroiusti, ${ }^{1}$ A Neri, ${ }^{1}$ G Somma, ${ }^{1}$ L Coppeta, ${ }^{1}$ I lavicoli, ${ }^{2}$ A Bergamaschi, ${ }^{2}$ A Magrini ${ }^{1}$
}

${ }^{1}$ Department of BiopathologyOccupational Medicine, Tor Vergata University, Rome, Italy; ${ }^{2}$ Institute of Occupational Medicine, Catholic University of the Sacred Heart, Rome, Italy

Correspondence to:

Antonio Pietroiusti, Department of Biopathology-Occupational Medicine, Tor Vergata

University, Via Montpellier 1, 00161 Rome, Italy; pietroiusti@ med.uniroma2.it

Accepted 19 July 2009 Published Online First 7 September 2009

\section{ABSTRACT}

Objective: Night-shift work is associated with ischaemic cardiovascular disorders. It is not currently known whether it may be causally linked to metabolic syndrome (MS), a risk condition for ischaemic cardiovascular disorders. The syndrome presents with visceral obesity associated with mild alterations in glucidic and lipidic homeostasis, and in blood pressure. The aim of this study was to assess whether a causal relationship exists between night-shift work and the development of MS. Methods: Male and female nurses performing night shifts, free from any component of MS at baseline, were evaluated annually for the development of the disorder during a 4-year follow-up. Male and female nurses performing daytime work only, visited during the same time period, represented the control group.

Results: The cumulative incidence of MS was $9.0 \%$ (36/402) among night-shift workers, and 1.8\% (6/336) among daytime workers (relative risk (RR) $5.0,95 \% \mathrm{Cl}$ -2.1 to 14.6). The annual rate of incidence of MS was $2.9 \%$ in night-shift workers and $0.5 \%$ in daytime workers. Kaplan-Meier survival curves of the two groups were significantly different (log-rank test; $p<0.001)$. Multiple Cox regression analysis (forward selection method based on likelihood ratio) showed that among selected variables lage, gender, smoking, alcohol intake, familiar history, physical activity, and work schedule) the only predictors of occurrence of MS were sedentariness (hazard ratio (HR) $2.92 ; 95 \% \mathrm{Cl} 1.64$ to $5.18 ; \mathrm{p}=0.017)$, and nightshift work (HR 5.10; 95\% Cl 2.15 to $12.11 ; p<0.001$ ).

Conclusions: The risk of developing MS is strongly associated with night-shift work in nurses. Medical counselling should be promptly instituted in night-shift workers with the syndrome, and in case of persistence or progression, a change in work schedule should be considered.

An association between shift work and coronary heart disease has been shown. ${ }^{1}$ In terms of prevention, however, it is more important to recognise susceptible individuals than those with overt disease.

In recent years, it has been suggested that metabolic syndrome (MS) (the association of even moderate degrees of visceral obesity, dyslipidaemia, abnormal blood pressure and serum glucose levels in the same individual) may sharply increase the risk of subsequent adverse coronary events; in particular, MS gives an almost twice increased risk for coronary heart disease, ${ }^{2}$ a two- to threefold increased risk for future ischaemic stroke ${ }^{3}$ and an even greater risk for diabetes. ${ }^{45}$

Workers involved in night shifts may be at increased risk for the development of the syndrome, on the basis of unfavourable effects of sleep

\section{What this paper adds}

- Night-shift work has been associated with metabolic syndrome, but no prospective study has been performed until now.

- Our prospective study shows that metabolic syndrome is causally linked to night shifts in healthcare workers.

- Changes in work schedule might be considered in workers showing a progression of the disorder in spite of dietary and lifestyle advice.

deprivation on the main components of the syndrome such as visceral obesity, ${ }^{67}$ blood pressure, $^{8}$ and insulin sensitivity. ${ }^{9}$ However, no prospective study has been performed until now to evaluate whether night-shift work actually represents a risk factor for the development of MS. The aim of the present study was therefore to evaluate whether the performance of night shifts may influence the incidence of MS in male and female nurses.

\section{METHODS}

Both male and female nurses were enrolled during an annual health surveillance programme performed by occupational physicians in three large hospitals (two teaching hospitals and one general hospital). The study started on January 2003 and ended on December 2007. A 4-year follow-up was planned for each included subject.

For the purpose of the present study, we considered as night-shift workers those on night shifts or rotating shifts that included at least an average of 4 nights per month during the year, and as daytime workers those regularly working between 07:00 and 21:00 from at least 1 year. At baseline, a standard medical history was collected and a physical examination was performed in all enrolled subjects. For the diagnosis of MS, appropriate measurements were performed during physical examination (abdominal girth and blood pressure), and venous blood was drawn for pertinent laboratory analyses (fasting glucose levels, serum triglycerides and high-density lipoprotein (HDL)-cholesterol). In detail, waist circumference was measured at the high point of the iliac crest at minimal respiration to the nearest $0.1 \mathrm{~cm}$ at the end of normal expiration. Two subsequent measurements of blood pressure were performed in all workers, and the mean of the two measurements was considered the actual value. Standard laboratory methods were used for serum parameters. Factors possibly affecting the prevalence of 
the syndrome such as smoking, alcohol intake, leisure time physical activity, and familiar history for any component of the syndrome or overt cardiovascular disease were obtained through specific questionnaires. Workers were defined as active smokers if they had smoked daily during the last 3 months before enrolment, and reported a daily habit of $>10$ cigarettes per day for at least 1 year during the past 10 years; they were considered as having a positive familiar history, if they reported at least one first-degree relative with a diagnosis of diabetes, hypertension, dyslipidaemia, coronary heart disease or ischaemic stroke under the age of 65 years. Alcohol intake was evaluated considering $110 \mathrm{~g}$ of alcohol per 11 of wine, $44 \mathrm{~g}$ of alcohol per 11 of beer, and $350 \mathrm{~g}$ of alcohol per 11 of spirit. Leisure time physical was investigated adapting a validated questionnaire, ${ }^{10}$ and classifying subjects' activity according to a previous report. ${ }^{11}$ Briefly, we calculated for each activity the weekly number of minutes spent in that activity. Total minutes per week spent in performing the activities were calculated by summing the weekly minutes for each activity. We divided the total weekly minutes into two categories: $<50$, and $\geqslant 50$ minutes/week of moderate to vigorous physical activity. ${ }^{11}$ Physical activities of light intensity were not reported. We defined "moderate" as the physical activity causing only light sweating or slight to moderate increase in breathing or heart rate when performed for at least 10 minutes (eg raking leaves, mowing the lawn or heavy cleaning); and "vigorous" as the activity causing heavy sweating, or significant increase in breathing or heart rate (eg running or fast bicycling). Workers spending $<50$ minutes/week in moderate physical activity were classified as sedentary.

Different criteria have been proposed by different institutions for the definition of MS. ${ }^{12-14}$ In the present study, we defined the syndrome according to the updated criteria of the National Cholesterol Education Program, ${ }^{14}$ because these criteria are simple to use in a clinical setting, have the advantage of avoiding emphasis on a single cause, and have been validated in a large number of studies. According to these criteria, MS is identified when $\geqslant 3$ of the following conditions are present in the same subject: waist $\geqslant 102 \mathrm{~cm}$ in men and $\geqslant 88 \mathrm{~cm}$ in women, serum triglycerides $\geqslant 1.695 \mathrm{mmol} / \mathrm{l}^{-1}(150 \mathrm{mg} / \mathrm{dl})$, HDL-cholesterol $<1.036 \mathrm{mmol} / \mathrm{l}^{-1}(40 \mathrm{mg} / \mathrm{dl})$ in men and $<1.295 \mathrm{mmol} / \mathrm{l}^{-1}(50 \mathrm{mg} / \mathrm{dl})$ in women, blood pressure $\geqslant 130$ or $\geqslant 85 \mathrm{mmHg}$ or treatment for hypertension, and plasma glucose $\geqslant 5.6 \mathrm{mmol} / \mathrm{l}^{-1}(100 \mathrm{mg} / \mathrm{dl})$.

For the purpose of the current study, participants taking active medications for any component of the syndrome were considered to be affected by that component of the syndrome.

Workers were included in the study if they had no trait of MS, no significant chronic disease possibly related with any component of the syndrome, and no change in work schedule during the 3 years preceding the inclusion in the study.

The Kaplan-Meier method and the log-rank test (MantelCox) were used to compare event-free survival among nightshift workers and daytime workers. With the use of proportional hazards analysis, hazard ratio (HR) and 95\% CI were generated for the unadjusted association between shift work or other baseline characteristics and the occurrence of MS. HRs were then adjusted for the confounding effects of other baseline characteristics, including age, sex, smoking status, alcohol intake, and familiar history of cardiovascular disease (Cox regression analysis). In addition, the assumption of the hazard proportionality of the Cox model was checked and the appropriate adjustment performed to obtain more robust estimates of the effects. In particular, Cox regression with time-dependent covariates was applied.
In the survival analysis, we "censored" all subjects for whom the duration of the study was limited in time for different reasons such as change in work or work schedule, the occurrence of significant diseases or death, the occurrence of pregnancy in females, and dropout during the follow-up period. Workers having $<1$ year of follow-up were excluded from the study.

Statistical analysis was performed with SPSS V.3.0. Student's t test and $\chi^{2}$ test were, respectively, used to compare continuous or categorical variables.

All the investigations performed in this study were conducted in accordance with the guidelines of the Declaration of Helsinki. Written consent from individuals had been granted, in accordance with the procedures approved by the ethical committee of our institution.

\section{RESULTS}

One thousand four hundred and sixty-four employees were eligible for baseline evaluation; among these, 1402 were actually visited (62 workers did not attend the scheduled baseline visit), and 664 were excluded for the following reasons: presence of at least one component of MS at baseline (478 workers), presence of significant chronic disorders possibly related to the syndrome (44 workers), change in work schedule during the preceding 3 years (82 workers), or work including $<4$ night shifts per month (four workers). Twenty-four eligible workers did not agree to participate in the study, and 32 additional workers were excluded because they had a follow-up of $<1$ year. Data are therefore referred to 738 workers. Among these, 402 were classified as night-shift workers and 336 as daytime workers.

The clinical characteristics of daytime and night-shift workers are shown in table 1.

There were no significant differences between the two populations in the frequency of risk factors for atherosclerosis. Traditional risk factors such as hypertension, diabetes, obesity and hyperlipidaemia were not included in the evaluation, since, as stated in the Methods, workers with these disorders were excluded from the study. However, baseline waist circumference data, although below the threshold for MS, are shown, considering the pivotal role of visceral obesity in the development of the syndrome.

The cumulative incidence of MS was $5.7 \%$ (42/738), with a total of 36 cases (9.0\%) occurring among the 402 night-shift workers, and with six cases (1.8\%) occurring among the 336 daytime workers (relative risk (RR) 5.0, 95\% C.I. 2.1 to 14.6). One hundred and fourteen subjects were censored: 36 because they changed their work or work schedule during the follow-up (24 among shift workers), 54 because they did not perform the

Table 1 Demographic, clinical characteristics of daytime workers and night-shift workers

\begin{tabular}{lcc}
\hline & $\begin{array}{l}\text { Daytime workers } \\
(\mathbf{n}=\mathbf{3 3 6})\end{array}$ & $\begin{array}{l}\text { Night-shift workers } \\
(\mathbf{n}=\mathbf{4 0 2})\end{array}$ \\
\hline Mean (SD) age, years & $37.9(8.5)$ & $38.9(8.4)$ \\
Male sex, $\mathrm{n}(\%)$ & $92(27.5)$ & $124(30.9)$ \\
Current smokers, $\mathrm{n}(\%)$ & $91(27.1)$ & $119(29.7)$ \\
Alcohol drinkers, $\mathrm{n}(\%)$ & $67(20.0)$ & $72(17.9)$ \\
Mean (SD) waist circumference, & $79.2(9.3)$ & $80.4(9.7)$ \\
$\mathrm{cm}$ & & $138(34.3)$ \\
Positive familiar history, $\dagger \mathrm{n}(\%)$ & $102(30.4)$ & $318(79.1)$ \\
Sedentariness, $\mathrm{n}(\%)$ & $255(77.1)$ &
\end{tabular}

*More than $30 \mathrm{~g} /$ day.

$\uparrow$ A positive familiar history refers to obesity, hypertension, hyperlipidaemia, diabetes, overt ischaemic cardiovascular disease, or a combination of the above. 


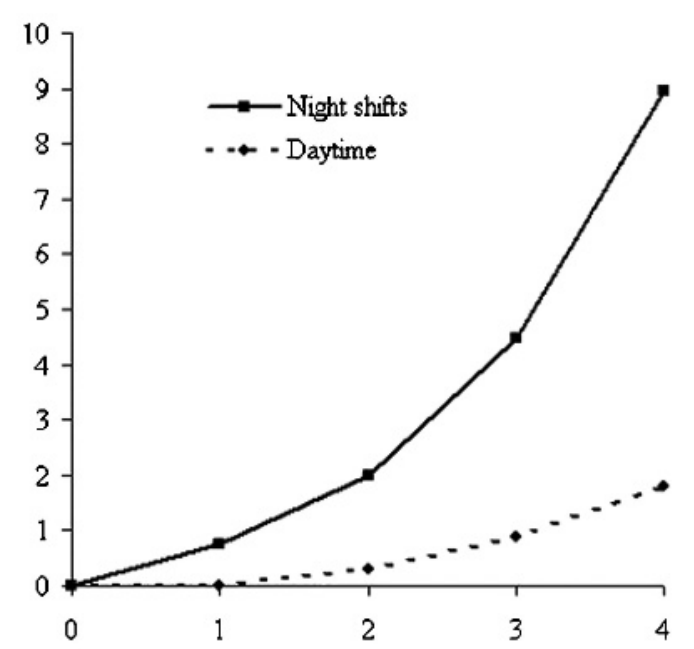

\section{N. at risk}

$\begin{array}{llllll}\text { Night shift } & 402 & 387 & 365 & 332 & 298 \\ \text { Duyytime } & 336 & 322 & 308 & 298 & 284\end{array}$

Figure 1 Cumulative incidence of metabolic syndrome in nightshift workers and daytime workers.

annual scheduled visit (32 among shift workers), 18 female nurses because they became pregnant (10 among shift workers), four because of a diagnosis of malignancy (two among nightshift workers), and two because they died for accidental reasons. Adjusting for the different length of follow-up until occurrence of the event/censoring, persons-year method allowed us to estimate that the annual rate of incidence of MS was $0.5 \%$ in daytime workers and $2.9 \%$ in night-shift workers. Kaplan-Meier survival curves of the two groups were significantly different (log-rank test; $\mathrm{p}<0.001$ ) (fig 1).

The cumulative incidence of the single components of the syndrome, shown in table 2, was similar for the two groups, except for visceral obesity, which was detected with increased frequency in night-shift workers (14.2\% vs $7.7 \%)$.

The combination of visceral obesity, low serum HDL and high serum triglycerides was by far the most common among subjects with MS, being detected in 22 out of 42 subjects $(52.4 \%)$.

Table 2 Cumulative incidence of metabolic risk factors among nightshift workers and daytime workers during the 4-year follow-up

\begin{tabular}{llll}
\hline Component & $\begin{array}{l}\text { Total population } \\
\text { (N= 738) }\end{array}$ & $\begin{array}{l}\text { Night-shift } \\
\text { workers }(\mathbf{n = 4 0 2 )}\end{array}$ & $\begin{array}{l}\text { Daytime workers } \\
\text { (n= 336) }\end{array}$ \\
\hline $\begin{array}{l}\text { Visceral obesity, n } \\
(\%)\end{array}$ & $83(11.2)$ & $57(14.2)$ & $26(7.7)$ \\
High BP, n (\%) & $85(11.5)$ & $45(11.2)$ & $40(11.9)$ \\
Low HDL-C, n (\%) & $89(12.1)$ & $56(13.9)$ & $33(9.8)$ \\
High TG, n (\%) & $89(12.1)$ & $51(12.7)$ & $38(11.3)$ \\
High glucose, n (\%) & $33(4.5)$ & $20(5.0)$ & $13(3.9)$ \\
\hline
\end{tabular}

Components by National Cholesterol Education Programme definition: visceral obesity: waist $\geqslant 102 \mathrm{~cm}$ in men and $\geqslant 88 \mathrm{~cm}$ in women; high BP: BP $\geqslant 130$ or $\geqslant 85 \mathrm{mmHg}$ or treatment for hypertension; low HDL-C: HDL-C $<1.036 \mathrm{mmol} / \mathrm{I}^{-1}(40 \mathrm{mg} / \mathrm{dl}$ ) in men and $<1.295 \mathrm{mmol} / \mathrm{I}^{-1}(50 \mathrm{mg} / \mathrm{dl})$ in women; high TG: serum triglycerides $\geqslant 1.695 \mathrm{mmol} / \mathrm{l}^{-1}(150 \mathrm{mg} / \mathrm{dl})$; high glucose: plasma glucose $\geqslant 5.6 \mathrm{mmol} / \mathrm{l}^{-1}$ (100 mg/dl).

BP, blood pressure; HDL-C, high-density lipoprotein-cholesterol; TG, triglyceride.
Table 3 Cox regression analysis for incidence of metabolic syndrome

\begin{tabular}{|c|c|c|c|c|}
\hline & \multicolumn{2}{|l|}{ Single-variable model } & \multicolumn{2}{|l|}{ Multi-variable model } \\
\hline & HR (95\% CI) & p Value & HR (95\% CI) & p Value \\
\hline Age & $0.97(0.94$ to 1.01$)$ & 0.14 & & \\
\hline Male sex & $1.16(0.61$ to 2.21$)$ & 0.64 & & \\
\hline Sedentariness & 2.96 (1.49 to 4.65$)$ & 0.01 & 2.92 (1.64 to 5.18$)$ & 0.02 \\
\hline Alcohol & 1.06 (0.49 to 2.30$)$ & 0.87 & & \\
\hline Smoking & $0.93(0.46$ to 1.84$)$ & 0.83 & & \\
\hline Family history & 0.83 (0.42 to 1.63$)$ & 0.60 & & \\
\hline Shift work & $5.19(2.19$ to 12.33$)$ & $<0.001$ & $5.10(2.15$ to 12.11$)$ & $<0.001$ \\
\hline
\end{tabular}

HR, hazard ratio.

After verifying the effect of each variable (age, gender, smoking, alcohol intake, familiar history, physical activity, and work schedule) separately on the occurrence of MS (table 3 - single-variable model), the first multiple Cox regression analysis was performed choosing a forward selection method based on likelihood ratio (table 3 - multi-variable model). This procedure indicated that the only predictors of occurrence of MS were sedentariness (HR 2.92; 95\% CI 1.64 to 5.18; $\mathrm{p}=0.017$ ), and night-shift work activity (HR 5.10; 95\% CI 2.15 to $12.11 ; p<0.001)$. No other variables entered the model (consistently, $p>0.20$ ). The interactions of age with sedentariness $(p>0.05)$ and with shift work $(p>0.20)$ tested to be nonsignificant. In our multi-variable model, the total number of events (occurrence of MS) was 42, and the considered variables were 7: one of interest (shift work) plus six relevant covariates. However, it has been suggested that the optimal event-pervariable ratio (EPV) for multi-variable follow-up studies should be at least $10,,^{15} 16$ whereas in our model it was $6(42 / 7)$. To overcome this problem, risk factors were summed in a single score (ranging between 0 and 5), substituting the five single factors. The previous finding was confirmed $(p<0.001$ for shift work and $p=0.24$ for risk factor score).

\section{DISCUSSION}

In this study, we show that in a population of relatively young workers free from any component of MS at baseline, the risk of developing the syndrome is strongly associated with the performance of night shifts.

Although several previous studies have suggested an association between shift work and MS, their cross-sectional design ${ }^{17-21}$ or retrospective nature ${ }^{22}{ }^{23}$ did not allow to evaluate the possible cause-effect relationship between these two conditions. To our knowledge, our study is the first with a prospective design, which gave us the opportunity to detect a causal association.

Our data confirm the central role of visceral obesity in determining the development of the syndrome, and the peculiar association of this component with shift work. ${ }^{17}$ On the other hand, obesity may be in turn related to sedentariness, which was present in a large proportion of our population, and was the only risk factor, in addition to shift work, predictive for the development of the syndrome. This finding is in agreement with recent data, showing an association between decreased physical activity and $\mathrm{MS},{ }^{24}$ and supports the recommendation of exercise training programmes in sedentary workers.

The mechanism by which night-shift work may induce MS was not investigated in the present study, and remains therefore speculative. Although lifestyle was similar in the two populations at baseline, we cannot confidently exclude changes during the follow-up period. In fact, formal questionnaires regarding, for example, leisure time physical activity, smoking or alcohol 
intake have not been administered during the follow-up examinations.

Independently of lifestyle, the disruption of the circadian rhythm may "per se" induce metabolic disturbances favouring the development of the syndrome, ${ }^{6-9}$ although it has been recently shown that increasing sleeping hours may also be associated with the syndrome. ${ }^{24}$ Thus, more than sleep deprivation, the irregular sleeping pattern associated with shift work may be crucial for explaining the observed association.

Furthermore, the stress linked to night-shift work might also play a role. In fact, an association of the syndrome with high levels of job stress has been reported, ${ }^{25}{ }^{26}$ and job stress is considered an important component of the association between shift work and cardiovascular disorders. ${ }^{27}$

The cumulative incidence of MS reported in the current study $(5.7 \%)$ is lower than that found in another study (about 33\% during a 3-year follow up period). ${ }^{28}$ In that study, however, all involved workers were of male sex, and therefore at increased risk for the syndrome, ${ }^{29}$ and subjects having one or two traits of the syndrome at baseline were included; furthermore, workers included in that study were of Asian ethnicity, and might have a different susceptibility in comparison to Caucasians. Our population was, however, composed almost exclusively of Caucasian people.

Our study has some limitations. First of all, our population was represented by healthcare workers, and therefore the findings cannot be generalised to other night-shift workers; furthermore, enrolled subjects were predominantly of female sex, and therefore the data regarding the male population are limited. On the other hand, the large participation to the study, involving almost all eligible workers, the very stringent selection criteria, allowing the inclusion only of subjects without any component of the syndrome, and the length of follow-up support the reliability of the data.

We feel that our findings may have a strong impact on health policy in shift workers; in fact MS may be present even with mild alterations of blood pressure, serum glucose and lipids, and body weight, whose clinical relevance may be easily overlooked if they are evaluated individually. Medical counselling should be promptly instituted in night-shift workers with the syndrome, and in case of persistence or progression, a change in work schedule should be considered.

\section{CONCLUSIONS}

This study adds to previous findings showing an association between night shifts and MS. It shows in fact for the first time that, during a 4-year period, the development of the syndrome is significantly higher in night-shift healthcare workers than in daytime healthcare workers, and suggests a causal relationship between the two events. These findings open the way to similar prospective confirmatory studies in workers involved in other activities, and suggest that appropriate medical surveillance and, if necessary, changes in work schedule should be considered in healthcare workers developing the syndrome.

Funding: The study was in part supported by a grant of Tor Vergata University (fondi Ateneo 60\% 2006).

Competing interests: None.

Ethics approval: Written consent from individuals had been granted, in accordance with the procedures approved by the ethical committee of our institution.

Patient consent: Obtained.

Provenance and peer review: Not commissioned; externally peer reviewed.

\section{REFERENCES}

1. Bøggild $\mathbf{H}$, Knutsson A. Shiftwork, risk factors and cardiovascular disease. Scand J Work Environ Health 1999;25:85-99.

2. Guize L, Pannier B, Thomas F, et al. Recent advances in metabolic syndrome and cardiovascular disease. (Review). Arch Cardiovascular Dis 2008;101:577-83.

3. Kurl S, Laukkanen JA, Niskanen L, et al. Metabolic syndrome and the risk of stroke in middle-aged men. Stroke 2006;37:806-11.

4. Hanley AJ, Karter AJ, Williams K, et al. Prediction of type 2 diabetes mellitus with alternative definitions of the metabolic syndrome: the Insulin Resistance Atherosclerosis Study. Circulation 2005;112:3713-21.

5. Yusuf S, Hawken S, Ounpuu S, et al. Effect of potentially modifiable risk factors associated with myocardial infarction in 52 countries (the INTERHEART study): casecontrol study. Lancet 2004;364:937-52.

6. Gangwisch JE, Malaspina D, Boden-Albala B, et al. Inadequate sleep as a risk factor for obesity: analyses of the NHANES I. Sleep 2005;28:1289-96.

7. Vorona RD,Winn MP, Babineau TW, et al. Overweight and obese patients in a primary care population report less sleep than patients with a normal body mass index. Arch Intern Med 2005;165:25-30.

8. Gangwisch JE, Heymsfield SB, Boden-Albala B, et al. Short sleep duration as a risk factor for hypertension: analyses of the first National Health and Nutrition Examination Survey. Hypertension 2006;47:833-9.

9. Gonzalez-Ortiz M, Martinez-Abundis E, Balcazar-Munoz BR, et al. Effect of sleep deprivation on insulin sensitivity and cortisol concentration in healthy subjects. Diabetes Nutr Metab 2000;13:80-3.

10. Centers for Disease Control and Prevention Codebook for Data Collection (1999-2000): Physical Activity Section of the SP and MEC CAPI Questionnaires. Available from: http:// www.cdc.gov/nchs/data/nhanes/frequency/paq.pdf (accessed 4 Feb 2003).

11. Pietroiusti A, Magrini A, Coppeta L, et al. Metabolic syndrome among operators using video display units in call centers. Scand J Work Environ Health Suppl 2007;3:49-53.

12. National Institutes of Health. National Institutes of Health: Third Report of the National Cholesterol Education Program Expert Panel on Detection, Evaluation and Treatment of High Blood Cholesterol in Adults (Adult Treatment Panel III). Executive Summary: National Institutes of Health, National Heart, Lung and Blood Institute, 2001 (NIH Publ. No. 01-3670).

13. Alberti KGMM, Zimmet PZ for the WHO Consultation. Definition, diagnosis and classification of diabetes mellitus and its complications. Part I diagnosis and classification of diabetes mellitus. Provisional report of a WHO consultation. Diabet Med 1998;15:539-53.

14. Grundy SM, Cleeman JI, Daniels SR, et al. Diagnosis and management of the metabolic syndrome: an American Heart Association/National Heart, Lung, and Blood Institute Scientific Statement. Circulation 2005:112:2735-52.

15. Peduzzi P, Concato J, Feinstein AR, et al. Importance of events per independent variable in proportional hazards regression analysis. II. Accuracy and precision of regression estimates. J Clin Epidemiol 1995;48:1503-10.

16. Concato J, Peduzzi P, Holford TR, et al. Importance of events per independent variable in proportional hazards analysis. I. Background, goals, and general strategy. J Clin Epidemiol 1995;48:1495-501.

17. Karlsson B, Knutsson A, Lindahl B. Is there an association between shift work and having a metabolic syndrome? Results from a population-based study of 27485 people. Occup Environ Med 2001;58:747-52.

18. Copertaro A, Bracci M, Barbaresi M, et al. Assessment of cardiovascular risk in shift healthcare workers. Eur J Cardiovasc Pre Rehabil 2008;15:224-9.

19. Di Lorenzo L, De Pergola G, Zocchetti C, et al. Effect of shift work on body mass index: results of a study performed in 319 glucose-tolerant men working in a southern Italian industry. Int J Obes Relat Metab Disord 2003;27:1353-8.

20. Ha M, Park J. Shift work and metabolic risk factors of cardiovascular disease. J Occup Health 2005;47:89-95.

21. Sookoian $\mathbf{S}$, Gemma $\mathrm{C}$, Fenànddez Giannotti $\mathrm{C}$, et al. Effects of rotating shift work on biomarkers of metabolic syndrome and inflammation. J Int Med 2007:261:285-92.

22. La Sala M, Pietroiusti A, Magrini A, et al. Metabolic syndrome and work. G Ital Med Lav Ergon 2007;29(3 Suppl):445-7.

23. Biggi N, Consonni D, Galluzzo V, et al. Metabolic syndrome in permanent night workers. Chronobiol Int 2008;25:443-54.

24. Santos A-C, Ebrahim S, Barros H. Alcohol intake, smoking, sleeping hours, physical activity and the metabolic syndrome. Prev Med 2007:44:328-34.

25. Kang MG, Koh SB, Cha BS, et al. Association between job stress on heart rate variability and metabolic syndrome in shipyard male workers. Yonsei Med J 2004;45:838-46.

26. Chandola T, Brunner E, Marmot M. Chronic stress at work and the metabolic syndrome. Br Med J 2006;332:521-5.

27. Tenkanen L, Sjöblom T, Kalimo R, et al. Shift work, occupation and coronary heart disease over 6 years of follow-up in the Helsinki heart study. Scand J Work Environ Health 1997;23:257-65.

28. Ryu S, Song J, Choi B-Y, et al. Incidence and risk factors for metabolic syndrome in Korean male workers, ages 30 to 39. Ann Epidemiol 2007;17:245-52.

29. Mattsson N, Rönnemaa T, Juonala M, et al. The prevalence of the metabolic syndrome in young adults. The cardiovascular risk in young Finns study. J Intern Med 2007;261:159-69. 